DOI: https://doi.org/10.46296/yc.v4i7edesp.0061

\title{
DISEÑO DE UN SISTEMA DE GESTIÓN DE CALIDAD CON LA APLICACIÓN DE LA NORMA ISO 9001: 2015 EN LA UNIDAD EDUCATIVA MARÍA AUXILIADORA DE MANTA
}

\section{DESIGN OF A QUALITY MANAGEMENT SYSTEM WITH THE APPLICATION OF ISO 9001: 2015 IN THE MARÍA AUXILIADORA EDUCATIONAL UNIT IN MANTA}

\author{
Solórzano-Murillo Estrella del Rocío ${ }^{1 *}$; Pinargote-Macías Eleticia Isabel ${ }^{2}$ \\ 1,2 Universidad Técnica de Manabí, UTM. Portoviejo, Ecuador. \\ *Correo: esolorzano4749@utm.edu.ec
}

\begin{abstract}
Resumen
El presente artículo tiene como objetivo exponer la construcción de un Sistema de gestión de la calidad con la aplicación de la norma ISO 9001:2015, en la Unidad educativa María Auxiliadora de Manta (UEMA). Las técnicas de recolección de datos utilizadas fueron encuestas y entrevistas dirigidas a los miembros de la comunidad educativa. La investigación parte desde análisis del contexto, teniendo como principales hallazgos que los padres de familia y estudiantes están conformes con los servicios de la institución, sin embargo, de acuerdo a los datos, la gestión de la calidad requiere perfeccionarse, se denota dificultades en comunicación e involucramiento de los clientes la toma de decisiones de la institución; por otro lado, el personal docente y administrativo que están en relación directa con estos procesos, comparte el criterio de implementar un sistema que garantice la calidad educativa. Así mismo, se evidenció debilidades en las fases del proceso administrativo como: planificación, organización y comunicación, lo que motivó a la puesta en marcha del diseño de un sistema de gestión con la Norma ISO 9001:2015 con el fin de mejorar la efectividad de los procesos, fortalecer el crecimiento institucional, la excelencia educativa y estar a la vanguardia de la educación del país.
\end{abstract}

Palabras clave: Calidad educativa; Norma ISO 9001:2015; sistema de gestión.

\begin{abstract}
This article aims to present the construction of a quality management system with the application of ISO 9001:2015, in the Maria Auxiliadora Educational Unit in Manta (UEMA). The data collection techniques used were surveys and interviews directed to members of the educational community. The research starts from the analysis of the context, having as main findings that parents and students are satisfied with the services of the institution, however, according to the data, the quality management requires improvement, there are difficulties in communication and involvement of customers in the decisions of the institution; on the other hand, teachers and administrative staff who are directly related to these processes, agree with the implementation of a system that ensures educational quality. Likewise, difficulties were evident in the phases of the administrative process such as: planning, organization and communication, which motivated the implementation of the design of a management system with ISO 9001:2015 in order to improve the effectiveness of the processes; strengthen institutional growth, educational excellence and be at the forefront of education in the country.
\end{abstract}

Keywords: Educational quality; ISO 9001:2015 standard; management system.

Información del manuscrito:

Fecha de recepción: 10 de septiembre de 2020.

Fecha de aceptación: 11 de noviembre de 2020.

Fecha de publicación: 16 de noviembre de 2020. 


\section{Introducción}

La calidad educativa es un tema que va tomando cada vez más posicionamiento en los procesos organizativos de las instituciones educativas. Éstas están en búsquedas continuos cambios que se generan en la gestión y vinculación en pro de mejorar la calidad de los servicios que ofrecen. Uno de los principales objetivos de las instituciones educativas es ofrecer una educación de calidad para el beneficio de la sociedad y la formación del futuro del país (Conde \& González, 2017, p.4), esta perspectiva, permite asumir a la calidad como un componente que estimula a las instituciones a implementar sistemas de mejoras integrales y funcionales.

Desde este contexto, el presente estudio propone implementar el Sistema de Calidad ISO 9001: 2015 en la Unidad Educativa Particular María Auxiliadora UEMA de la ciudad de Manta, la misma que en su visión, plantea alcanzar en sus servicios la excelencia académica.

El sistema de gestión de calidad es una evaluación aplicada a la gestión que se desarrolla en una institución y tiene como finalidad la organización de un adecuado proceso productivo. Para Martínez, Laguado \& Flórez (2018), un Sistema de Gestión de Calidad (SGC) se define como "la articulación de métodos, recursos, personas e insumos que interactúan en el uso racional de los recursos y que tiene como propósito la consecución de la satisfacción de los clientes" (pp.217).

Las ISO son normas para certificaciones internacionales de calidad, que una empresa puede obtener por organismos certificadores, los cuales acreditan la capacidad que tiene la empresa 0 institución a fin de ofrecer un producto o servicio que cumpla los requisitos del cliente.

Los sistemas de gestión de calidad basados en la norma ISO 9001:2015, permiten mejorar la gestión de procesos administrativos, y sustantivos a la docencia, lo cual supone un aumento de productividad y motivación en cada uno de los actores involucrados (Fontalvo \& $\mathrm{Hoz}, 2008$ ).

Así, con la implementación de la norma, la UEMA podrá obtener mayores ventajas competitivas en 
comunicación, estandarización, y mejora de los procesos con respecto a otras instituciones particulares de la ciudad, lo que permite abrir caminos sólidos a la productividad y a la competitividad, de esta manera se contribuye al fortalecimiento de la gestión pedagógica y administrativa encaminada a innovar y mejorar la acción educativa.

En la actualidad, la Unidad Educativa María Auxiliadora no cuenta con un sistema de gestión de la calidad basado en normas internacionalizadas, se pretende a través de una gestión apropiada con estándares establecidos, analizar los aspectos administrativos-educativos mejorar la calidad del servicio lo que garantizará contar con una declaración documentada del desarrollo de actividades sistematizadas con el debido monitoreo para garantizar el efectivo tratamiento del entorno administrativo y asegurar la real organización, operación, control de sus acciones y registros.

El objetivo de educar con calidad implica no solo que el profesor debe dominar un tema, y que para asumir la responsabilidad debe tener cualidades personales y académicas idóneas, sino que las dinámicas de clases, evaluaciones y de seguimiento de los alumnos garanticen el alcance de los objetivos institucionales propuestos, entre los que se destaca llenar las expectativas de los estudiantes y padres de familia a partir del cumplimiento de indicadores de metas. (Díaz, T, 2009)

Desde esta mirada, se delimita como objetivo de este trabajo el diseño de un sistema de gestión de calidad basado en la norma ISO 9001:2015 en la unidad educativa María Auxiliadora de Manta, para contribuir al mejoramiento y efectividad de sus servicios.

\section{Metodología}

La investigación aborda la problemática desde enfoques descriptivos, biográficos, documentales y de campo; el análisis de la realidad se enmarca en las debilidades y potencialidades que puedan encontrarse en el contexto, además desde la revisión y estudio de documentación oficial de la Norma ISO, recolectar elementos para el diseño de la propuesta de 
implementación, la que se relaciona directamente con la participación en el campo educativo. La investigación está estructurada en dos etapas: la primera donde se realiza la parte diagnóstica descriptiva y la búsqueda de información bibliográfica; la recopilación de datos y el empleo de técnicas secundarias como análisis de artículos científicos, revistas indexadas en bases científicas, secciones de libros y normas internacionales que ayudan a comprender el enfoque de la gestión de calidad en la educación; y la segunda de campo donde se aplicó una encuesta a los involucrados internos y externos con una serie de preguntas cuyo objetivo es conocer los criterios en relación a las acciones que se llevan a cabo dentro de la institución, también se adiciona una entrevista a la autoridad principal con la finalidad de tener un enfoque cualitativo desde la concepción de la gestión gerencial.

En el grupo se consideró la población compuesta por estudiantes, administradores, maestros y padres; Para el universo de padres y estudiantes se aplicó el cálculo de tamaño de muestra, escogiéndose 140 estudiantes y padres de familia, a todos los miembros administrativos $y$ docentes de la institución. La información obtenida, permitió interpretar y comparar información sobre la satisfacción del cliente, la calidad y la gestión de la educación.

\section{Resultados}

A continuación, se expondrán los principales resultados de la investigación.

Resultados de las encuestas: Las encuestas aplicadas a los padres de familia, estudiantes y al personal docente y administrativo, quienes están en contacto directo con los procesos de la institución.

Gráfico 1. Servicio que recibe por parte del personal docente y administrativo.

Fuente: Encuesta a padres de familia y estudiantes.

Elaboración propia a partir de los resultados obtenidos.

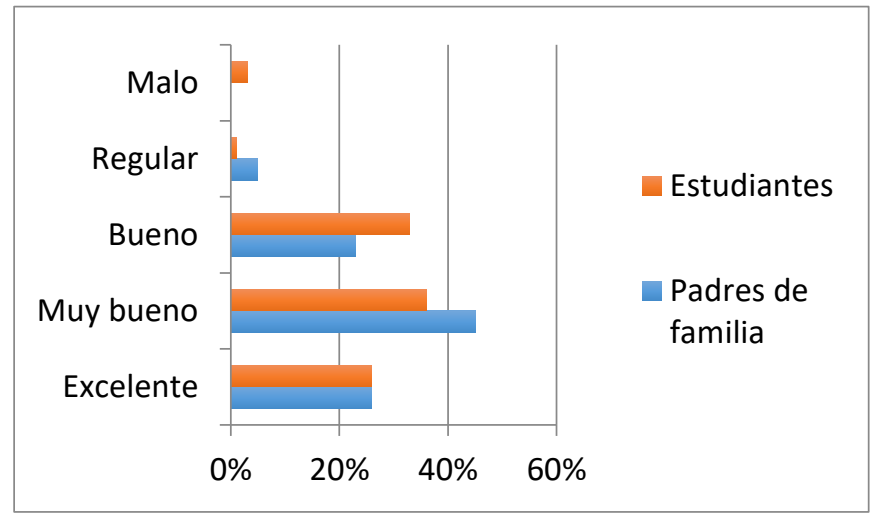

Como se observan los resultados el $36 \%$ de estudiantes y el $45 \%$ de 
padres de familia están de acuerdo con que el servicio prestado por la institución considerándolo, muy bueno; por lo que se puede deducir que en su mayoría los clientes de la institución están satisfechos por los servicios, aunque existe un margen de posibilidad de poder mejorar la atención y satisfacción del cliente para acrecentar la calidad educativa.

Gráfico 2. La opinión de los clientes en los procesos.

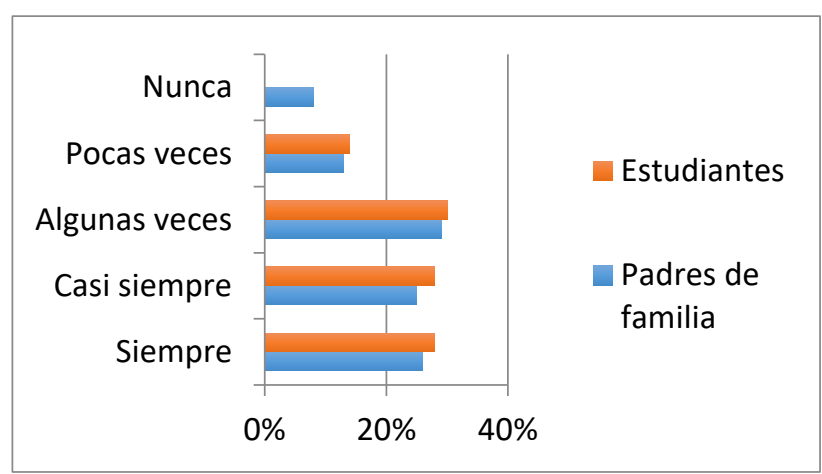

Fuente: Encuesta a padres de familia y estudiantes.

Elaboración propia a partir de los resultados obtenidos.

Los clientes de la institución indican que sus opiniones son escuchadas y tomadas en cuenta en los procesos que garantizan calidad, sin embargo, el margen en el que se encuentran estas opiniones en porcentajes está muy cercanas (oscilan entre el $25 \mathrm{y}$ $30 \%$ ) las opciones algunas veces, casi siempre y siempre, por lo que se podría deducir que existen clientes que se involucran más en los procesos que otros, al menos lo perciben así.

Gráfica 3. Cumplimiento de expectativas institucionales

\begin{tabular}{|r|r|r|}
\hline Nunca & \\
Pocas veces & & \\
Algunas veces \\
Casi siempre \\
Siempre
\end{tabular}

Fuente: Encuesta a padres de familia. Elaboración propia a partir de los resultados obtenidos.

Respecto a las expectativas del servicio que brinda la unidad educativa, los estudiantes y padres de familia indican que estas casi siempre se cumplen. Lo que lleva a derivar que los involucrados están satisfechos con la atención que reciben, no obstante, es necesario que la institución se encamine a cumplir los estándares que exigen cambios en esta era globalizada.

Gráfico 4. La implementación de un sistema de gestión de calidad desde la perspectiva del cliente.

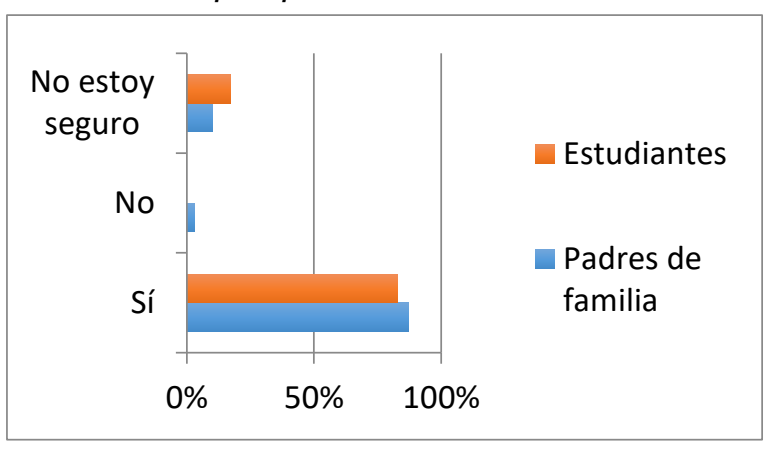

Fuente: Encuesta a padres de familia y estudiantes.

Elaboración propia a partir de los resultados obtenidos. 
En relación con la categoría: necesidad de implementar un sistema de gestión de calidad se observa que $87 \%$ de padres de familia están de acuerdo con esta iniciativa, al igual que el $83 \%$ del estudiantado, por lo que se deduce que existe una actitud positiva frente a los cambios que la gestión que se quieren implementar en la institución ya que en muchas ocasiones las normas direccionadas por el Ministerio de Educación no permiten alcanzar un óptimo nivel de servicio educativo.

Gráfico 5. Participación del personal docente y administrativo en la toma de decisiones para las mejoras del servicio.

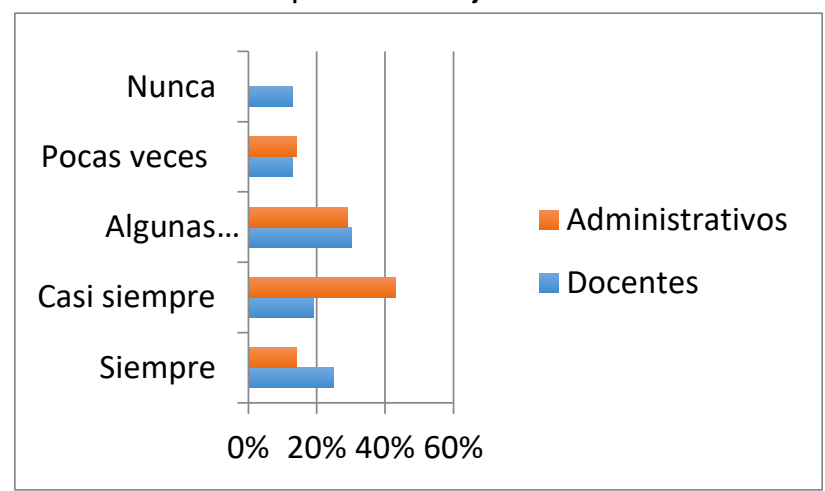

Fuente: Encuesta a padres de familia y estudiantes.

Elaboración propia a partir de los resultados obtenidos.

En esta pregunta las opiniones son diversas, por un lado, los docentes en un $30 \%$ indican que se toma en consideración su opinión para mejoras algunas veces, sin embargo, el $70 \%$ restante tiene opiniones divididas con valores muy cercanos, por ejemplo, el 13\% anuncia que nunca son tomados en cuenta y un $25 \%$ que siempre están involucrados. Lo que resulta a interpretar es que los docentes se involucran en distintos niveles, y esto puede relacionarse a las responsabilidades asignadas, ya que un docente que es tutor de aula se encuentra más involucrado en los procesos. Se considera necesario que la institución adopte medidas idóneas que den lugar a la interacción de los distintos departamentos en la toma de decisiones que conlleven a una misma finalidad de brindar educación de calidad y calidez.

Gráfico 6. Medios que aporta la institución para prestar servicios.

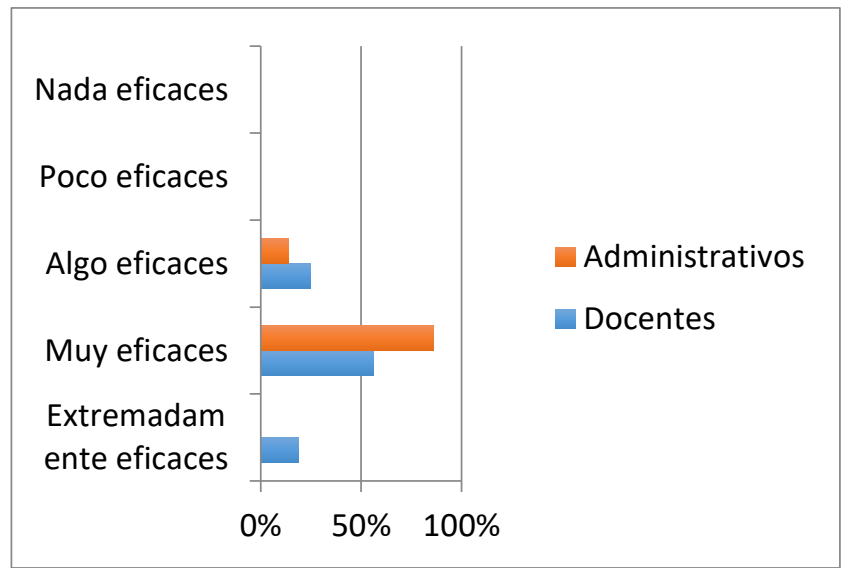

Fuente: Encuesta a padres de familia y estudiantes.

Elaboración propia a partir de los resultados obtenidos.

En el gráfico 6 , se puede evidenciar que el personal administrativo se 
siente casi en su totalidad conforme con los medios que provee la institución para prestar sus servicios; empero, para algunos de estos medios son algo eficientes, datos que se pueden interpretar desde la subjetividad o el involucramiento que tienen los docentes en los procesos. Por tanto, requiere que la institución diseñe planes de acción donde haya la participación activa de todo el claustro.

Gráfico 7. Consideraciones para la implementación un sistema de gestión.

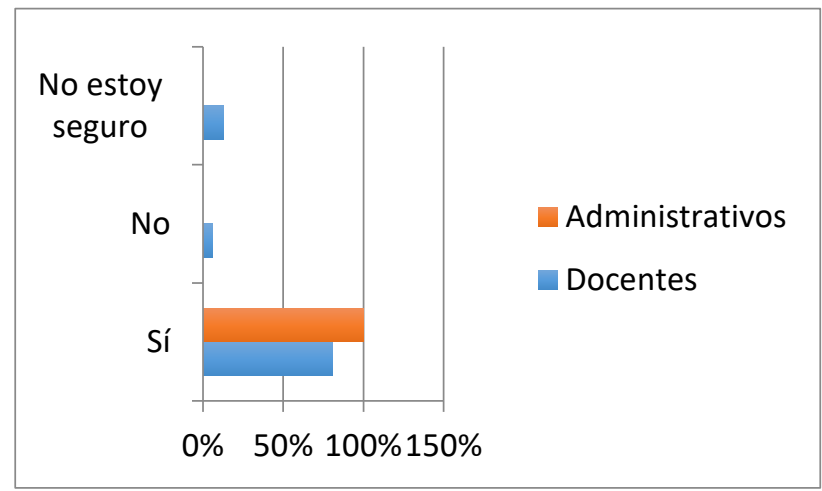

Fuente: Encuesta a padres de familia y estudiantes.

Elaboración propia a partir de los resultados obtenidos.

El personal administrativo considera en un $100 \%$ que sería necesario implementar un sistema de gestión en la institución, mientras que los docentes están de acuerdo en un $81 \%$, Estos datos revelan el interés de quienes conforman el equipo de trabajo de la institución por perfeccionar los procesos, quienes están conscientes que al aplicar un sistema de gestión se puede fortalecer calidad de los servicios que ésta ofrece y por ende mantener la satisfacción de todos quienes conforman la unidad educativa.

- Resultados de la entrevista: La entrevista fue aplicada a la rectora de la Unidad Educativa María Auxiliadora, encargada de la dirección de esta institución. A continuación, se exponen desde las categorías de análisis, las principales respuestas.

- Categoría:

Procesos institucionales y responsabilidades asumidas

“...Evaluar, impulsar y controlar el buen funcionamiento de la institución tanto en desarrollo enseñanza- aprendizaje, como en formar espiritualidad salesiana basada en valores y el sistema preventivo de Don Bosco."

Con relación del diseño del plan de trabajo

“...Por supuesto nos regimos con un plan anual direccionado con todos los lineamientos que 
emite el Ministerio de Educación, además de Códigos de convivencia internos para mejorar la calidad y calidez educativa."

Procesos de elaboración del Plan Anual de Trabajo

“...Se parte desde un análisis FODA para luego en conjunto con vicerrectorado y consejo ejecutivo organizar el plan anual, el mismo que es socializado con docentes y reforzado teniendo en consideración sus sugerencias antes de ser llevado a la práctica."

En cuanto a los objetivos institucionales,

“...son: ser protagonistas del aprendizaje y formación humano- cristiano, profesional y vocacional, con el desarrollo de la capacidad de liderazgo y animación expresada en el trabajo cooperativo y servicio de los demás.

Ser conscientes del valor de sí mismo, cultivar el crecimiento de libertad y responsabilidad, con una visión abierta y optimista de la realidad cotidiana, aceptar y valorar con autenticidad la riqueza de la diversidad."

- Categoría: Calidad Educativa.

Capacitaciones al personal docente y administrativo.

“...Las capacitaciones son periódicas basadas en los lineamientos que solicite el Ministerio de Educación, además de las emitidas por la Congregación de Hermanas Salesianas Hijas de María Auxiliadora. Estas capacitaciones van desde fortalecer y actualizar conocimientos, inclusión a la institución estudiantes con necesidades educativas especiales, espiritualidad salesiana, actualizaciones de planificaciones y todo lo que se considere necesario en conjunto a la normativa vigente y resolución ministerial y congregación."

- Categoría: Gestión de calidad.

En cuanto a gestión administrativa de la Unidad Educativa: ejecución, seguimiento y monitoreo de la gestión administrativa

"...Se crean comisiones y dimensiones que se encargan del seguimiento, monitoreo en conjunto 
con DECE y trabajo social siempre se analiza si se están logrando los objetivos."

Interés por implementar un Sistema de Gestión de Calidad en la institución

“...Sí, porque esto fortalecería a la institución y ayudaría a alcanzar de mejor manera sus objetivos y mantener la continuidad de los mejores puestos a nivel nacional y a futuro poder integrar el bachillerato internacional."

\section{Discusión}

Los resultados expedidos en las encuestas aplicadas a estudiantes y padres de familia dieron lugar al siguiente análisis:

El servicio que los clientes reciben por parte del personal docente y administrativo es considerado muy bueno (gráficos 1), lo que significa que la satisfacción es alta, pero podría llegar a la excelencia, citando Aceves (2003), la empresa debe asumir la tarea de comprender los aspectos estratégicos de la calidad de la atención al cliente para que pueda realizar correctamente los servicios y ampliar su base de clientes, esto se debe al establecimiento de una imagen más optimizada y posterior incorporación de sugerencias. (pp.2).

La necesidad de involucrar a los clientes y al personal en los procesos de gestión, resulta esencial, los clientes están de acuerdo que sus opiniones "algunas veces" es solicitada, cuando lo ideal sería siempre se sientan parte de la toma de decisiones institucionales. De acuerdo con Graham (2016), convertirse en un referente de los servicios del mercado requiere identificar puntos clave para los clientes, identificar desviaciones de las expectativas a través de la voz de los clientes, y continuar tomando acciones para brindar una experiencia inolvidable de las emociones positivas de los clientes, pues se sabe que el cliente es la base de la empresa (pp. 7).

Otro dato relevante es que, en su gran mayoría de la comunidad educativa, constituida por padres de familia, estudiantes, personal docente y administrativo consideran oportuno adoptar un sistema de gestión de calidad que fortalezca la dinámica e interacción institucional. 
Sin duda, la adopción de un modelo donde se estandaricen criterios para evaluar la calidad educativa sería una medida pertinente para estar a la vanguardia y a las exigencias de la época.

La puesta en marcha un sistema basado en la norma ISO 9001: 2015, podría ayudar a que los resultados de satisfacción del cliente y de los miembros de la institución mejoren, alcanzando estándares de excelencia. Entre los beneficios de implementar un sistema de gestión basado en la norma son: capacidad de proporcionar regularmente productos y servicios que cumplan con los requisitos de los clientes, las leyes y regulaciones aplicables aumentando la satisfacción del cliente y conocer los riesgos y oportunidades relacionados con sus antecedentes y objetivos. (Norma ISO 9001, 2015).

\section{Conclusiones}

El análisis de los resultados llevó a las siguientes generalizaciones:

El criterio, opiniones, actitudes y expectativas de los involucrados servirá de norte para la toma de decisiones que coadyuven a satisfacer las expectativas de la comunidad educativa y las demandas de la sociedad.

Implementar y certificar un sistema de gestión de la calidad de acuerdo con la norma ISO 900, constituye un gran desafío, esto significa adoptar un nuevo concepto y hacer un cambio profundo en la cultura de trabajo, por lo que requiere una intervención insondable de todos los involucrados.

En este proceso es crucial considerar el rol que juegan tanto los docentes como los estudiantes, así como el manejo de una comunicación horizontal a fin de asegurar la participación activa de los miembros de la comunidad con miras de conducir a la institución al camino de la excelencia.

\section{Bibliografía}

Aceves, J. (2013). Importancia de la calidad del servicio al cliente para el funcionamiento de las empresas. Revista el buzón de Pacioli, 3-21.

Conde, M. D. D., \& González, M. R. F. (2018). Educación social y escuela en España. A propósito de la formación e 
inserción laboral. Revista iberoamericana de Educación, 76, 209-228.

Díaz, T. (2009). Excelencia académica y formación integral. Centro Editorial Universidad del Rosario. I edición.

Fontalvo, T., \& Hoz, E. D. (2018). Diseño e Implementación de un Sistema de Gestión de la Calidad ISO 9001:2015 en una Universidad Colombiana (Vol. 11). Cartagena: Scielo.

Guzmán Aguilar, C. (2012). Estado del Arte de los Sistemas de Gestión de la Calidad y del Medio Ambiente (Tesis de Maestría) [versión electrónica]. Universidad de Sevilla, Sevilla, España. Recuperado desde http://bibing.us.es/proyectos/a breproy/70382/fichero/TFM.+ PARTE+I.+Estado+del+Arte+ de+los+Sistemas+de+Gesti\% C3\%B3n+de+.pdf

Graham, C. (2016). Una buena experiencia al cliente tiene un impacto financiero positivo. Deloitte (Vol.1). Colombia: DEC.

Martínez-Rojas, A. B., LaguadoRamírez, R. I., \& FlórezSerrano, E. G. (2018). Factores de éxito de la certificación ISO 9001 en empresas de Cúcuta y su Área Metropolitana. Estudios
Gerenciales, 34(147), 216228.

Norma Internacional ISO/DIS 9001:2015, Ginebra, Suiza: Manual. 